\title{
AN ALGORITHM FOR EXTRACTING THE GEOMETRIC PARAMETERS OF A RIGHT CIRCULAR CONE FROM THE COEFFICIENTS OF ITS ALGEBRAIC EQUATION
}

\author{
Mohamed Ali Said \\ Math. and Physics Dept., Faculty of Eng., Zagazig Univ., Egypt \\ mmamsaid@hotmail.com
}

\begin{abstract}
A right circular cone is an important geometric primitive that is used in many applications. The geometric parameters of the cone are the coordinates of its vertex, the direction of its axis, and the semi-angle of its vertex. These geometric parameters do not appear explicitly in the cone's algebraic equation which is widely used because of its simplicity. So, in this paper an algorithm is introduced to extract the cone's geometric parameters from the coefficients of its algebraic equation. A solid theoretical frame work is given through three propositions. The algorithm is tested on an eleven cases and proves reliability and simplicity. The algorithm can also detect non right circular cone cases.
\end{abstract}

Key Words: Cone, geometric parameters, algorithm. MSC2010: 97G70, 65D18, 68U05, $68 W 99$.

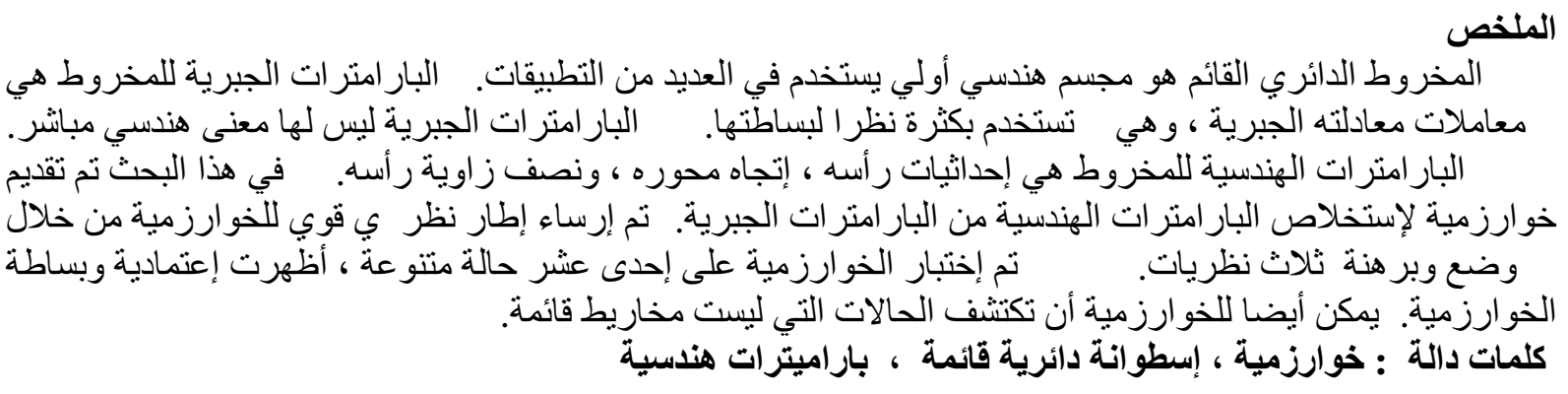

\section{INTRODUCTION}

A circular cone is one of the most important geometric primitives used in applications; a 95\% of industrial objects can be described by spheres, planes, cones, cylinders, and tori [1].

A right circular cone is a cone whose cross-section, by a plane perpendicular to its axis, is a circle.

The geometric parameters of a right circular cone are: the vertex coordinates $\left(x_{0}, y_{0}, z_{0}\right)$, the direction of the axis $(\mathbf{C}, \boldsymbol{G}, \mathbf{0}$, and the vertex semi-angle $\boldsymbol{C}$

The geometric equation of a right circular cone is given in $[2,3]$ as

$\frac{\left[\lambda\left(x-x_{0}\right)+\mu\left(y-y_{0}\right)+v\left(z-z_{0}\right)\right]^{2}}{\left[\left(x-x_{0}\right)^{2}+\left[y-y_{0}\right)^{2}+\left(z-z_{0}\right)^{2}\right]\left(\lambda^{2}+\mu^{2}+v^{2}\right)}=\cos ^{2} \alpha$

While the algebraic equation of a right circular cone as a quadric surface is given in [4] as

$A x^{2}+B y^{2}+C z^{2}+D x y+E x z+F y z+G x+H y+I z+J=0$

The conditions, under which equation (2) represents a right circular cone, are given in [5]. 
The cones are still live in research and attract attention in different research directions. Fitting of cones to data $[6,7,8,9,10]$. Extraction of cones from minimal point-sets [11]. Cone identification [12]. Geometric modeling [13].

The problem addressed in this paper is how to extract the geometric parameters of a right circular cone from the coefficients of its algebraic equation.

The remaining of the paper is arranged as follows: in section 2, a comparison is made between the coefficients of the algebraic and geometric equations. In section 3 , the extraction of the vertex coordinates is given. In section 4, extraction of the axis direction is introduced. In section 5, the semi-angle of the vertex is extracted. In section 6, the proposed algorithm is introduced. In section 7, the algorithm is tested on eleven cases. Finally, section 8 is devoted for conclusion.

\section{COMPARING COEFFICIENTS OF THE CONE'S ALGEBRAIC AND GEOMETRIC EQUATIONS}

$\lambda^{2}+\mu^{\text {IIT }}$

Expanding the geometric equation, the result is

Comparing the coefficients of equations (2) and (3), the result is

$$
\begin{aligned}
& \left.A=\lambda^{2}-\lambda^{2}+\mu^{2}+v^{2}\right) \cos ^{2} \alpha \\
& \left.B=\mu^{2}-\lambda^{2}+\mu^{2}+v^{2}\right) \cos ^{2} \alpha \\
& \left.C=v^{2}-\lambda^{2}+\mu^{2}+v^{2}\right) \cos ^{2} \alpha \\
& D=2 \lambda \mu \\
& E=2 \lambda v \\
& F=2 \mu v \\
& \left.G=-2\left[\lambda^{2}-\lambda^{2}+\mu^{2}+v^{2}\right) \cos ^{2} \alpha\right] x_{0}-2 \lambda \mu y_{0}-2 \lambda v z_{0} \\
& \left.H=-2 \lambda \mu x_{0}-2\left[\mu^{2}-\lambda^{2}+\mu^{2}+v^{2}\right) \cos ^{2} \alpha\right] y_{0}-2 \mu v z_{0} \\
& \left.I=-2 \lambda v x_{0}-2 \mu v y_{0}-2\left[v^{2}-\lambda^{2}+\mu^{2}+v^{2}\right) \cos ^{2} \alpha\right] z_{0} \\
& \left.I=\left[\lambda^{2}-\left(\lambda^{2}+\mu^{2}+v^{2}\right) \cos ^{2} \alpha\right] x_{0}^{2}+\left[\mu^{2}-\left(\lambda^{2}+\mu^{2}+v^{2}\right) \cos ^{2} \alpha\right] y_{0}^{2}+\left[v^{2}-\alpha^{2}+\mu^{2}+v^{2}\right) \cos ^{2} \alpha\right] z_{0}^{2}+\left(2 \lambda_{i}\right.
\end{aligned}
$$

\section{EXTRACTING THE VERTEX COORDINATES; $\left(x_{0}, y_{0}, z_{0}\right)$}

Solving equations (10), (11), and (12) simultaneously for $x_{0}, y_{0}$, and $z_{0}$ using Cramer's rule, the result is

$$
x_{0}=\frac{\Delta_{1}}{\Delta}, \quad y_{0}=\frac{\Delta_{2}}{\Delta}, \quad z_{0}=\frac{\Delta_{3}}{\Delta}
$$

where

$$
\Delta=\left|\begin{array}{ccc}
2 A & D & E \\
D & 2 B & F \\
E & F & 2 C
\end{array}\right| \neq 0 \quad, \quad \mathbf{\Delta}_{\mathbf{1}}=\left|\begin{array}{ccc}
-G & D & E \\
-H & 2 B & F \\
-I & F & 2 C
\end{array}\right|
$$


$\mathbf{\Delta}_{\mathbf{2}}=\left|\begin{array}{ccc}2 A & -G & E \\ D & -H & F \\ E & -I & 2 C\end{array}\right|, \quad \mathbf{\Delta}_{\mathbf{3}}=\left|\begin{array}{ccc}2 A & D & -G \\ D & 2 B & -H \\ E & F & -I\end{array}\right|$

If $\Delta=0$ then the surface is not a cone.

\section{Extracting the Direction of the Axis; $\left.\begin{array}{lll}\lambda_{z} & \mu_{r} & v\end{array}\right)$}

Cases where the cone-axis is parallel to one of the principal axes is treated in the following proposition.

Proposition (4.1):

For the right circular cone $A x^{2}+B y^{2}+C z^{2}+D x y+E x z+F y z+G x+H y+I z+J=0$

with axis direction $\lambda_{2} \quad \mu_{r} \quad w$, let $D=E=F=0$ and $A B C>0$ then

Case 1:

If $A=B$ and $C>0$ then the cone-axis is parallel to $z$-axis with $\lambda=0, \mu=0$,

$v= \pm \sqrt{C-A}$. If $C \neq 0$ then the cone's equation is multiplied by (-1) and the resulting

coefficients are used.

Case 2:

If $A=C$ and $B>0$ then the cone-axis is parallel to $y$-axis with $\lambda=0, \mu= \pm \sqrt{B-C}$, $v=0$. If $B \ngtr 0$ then the cone's equation is multiplied by (-1) and the resulting coefficients are used.

Case 3:

If $B=C$ and $A>0$ then the cone-axis is parallel to $x$-axis with $\lambda= \pm \sqrt{A-C}, \mu=0$,

$v=0$. If $A \neq 0$ then the cone's equation is multiplied by (-1) and the resulting coefficients are used.

Proof:

Case 1:

Substitute by $D=E=F=\mathbf{0}$ in equations (7-9) then

$\lambda \mu=0, \lambda v=0, \mu v=0$

Since $\lambda, \mu$, and $v$ can't be all zeros in the same time, then there are 3 possible solutions:

$\{\lambda=\mu=0, v \neq 0\},\{\lambda=v=0, \mu \neq 0\}$, or $\{\mu=v=0, \lambda \neq 0\}$

Set $A=B$ in equations (4- 5) implies

$\lambda^{2}=\mu^{2} \Rightarrow \lambda= \pm \mu$

Substitute from equation (19) in equations (17) then

$\pm \mu^{2}=0, \pm \mu \nu=0, \mu v=0 \Rightarrow \mu=0 \Rightarrow \lambda=0$

Using equations (20) in equations $(4-6) \Rightarrow$

$A=-v^{2} \cos ^{2} \alpha$

$B=-v^{2} \cos ^{2} \alpha$

$C=v^{2}-v^{2} \cos ^{2} \alpha=v^{2}\left(1-\cos ^{2} \alpha\right)=v^{2} \sin ^{2} \alpha$

Using equations (21-23)

$A B C>0 \Rightarrow v^{6} \cos ^{4} \alpha \sin ^{2} \alpha>0 \Rightarrow v \neq 0$

Using equations (21-23) then

$v^{2}=C-A=C-B \Rightarrow v= \pm \sqrt{C-A}$

Thus the cone axis is parallel to z-axis with $\lambda=0, \mu=0, v= \pm \sqrt{C-A}$, where $C>0$. 
Proofs of case 2 and case 3 are similar to that of case 1

Note (4.1): if the condition $A B C>0$ is not satisfied, then multiply the cone's equation by $(-1)$ and use the resulting coefficients.

Note (4.2): without loss of generality, the positive root can be used in the three cases mentioned in propositions (4.1).

Cases where the cone-axis is parallel to one of the principal planes is treated in the following proposition.

\section{Proposition (4.2):}

For the right circular cone $A x^{2}+B y^{2}+C z^{2}+D x y+E x z+F y z+G x+H y+I z+J=0$, let the axis direction be $\boldsymbol{\Lambda}_{v} \quad \mu_{v} \quad v$ then

Case 1:

If $D \neq 0, E=0, F=0$, and $C<0$ then the cone-axis is parallel to the $x y$-plane with $\lambda=\sqrt{A-C}$, $\mu=\operatorname{sign}(B) \sqrt{B-C}$, and $v=0$. If $C * 0$ then the cone's equation is multiplied by (-1) and the resulting coefficients are used.

Case 2:

If $D=0, E \neq 0, F=0$, and $B<0$ then the cone-axis is parallel to the xz-plane with $\lambda=\sqrt{A-B}, \mu=0$, and $v=\operatorname{sign}(E) \sqrt{C-B}$. If $B \neq 0$ then the cone's equation is multiplied by (-1) and the resulting coefficients are used.

Case 3:

If $D=0, E=0, F \neq 0$, and $A \leqslant 0$ then the cone-axis is parallel to the yz-plane with $\lambda=0$, $\mu=\sqrt{B-A}$, and $v=\operatorname{sign}(F) \sqrt{C-A}$. If $A \neq 0$ then the cone's equation is multiplied by $(-1)$ and the resulting coefficients are used.

\section{Proof:}

Case1:

Substitute $\mathrm{D} \neq 0, \mathrm{E}=0$, and $\mathrm{F}=0$ in equations $(7-9) \Rightarrow$

$\lambda \mu \neq 0$ )

$\lambda \nu=\mathbf{0}\}$

$\mu \nu=0\} \Rightarrow \lambda \neq 0, \mu \neq 0$, and $v=0$

$\Rightarrow$ the cone-axis is parallel to the xy-plane.

Substitute $v=0$ in equations (4-6) gives

$$
\left.\begin{array}{c}
A=\lambda^{2}-\left(\lambda^{2}+\mu^{2}\right) \cos ^{2} \alpha \\
B=\mu^{2}-\left(\lambda^{2}+\mu^{2}\right) \cos ^{2} \alpha \\
C=-\left(\lambda^{2}+\mu^{2}\right) \cos ^{2} \alpha
\end{array}\right\}
$$

$\Rightarrow \mathrm{C}$ is negative, otherwise multiply the cone equation by $(-1)$.

Also, $A-C=\lambda^{2}$ and $B-C=\mu^{2}$.

$\lambda$ and $\mu$ should satisfy $D=2 \lambda \mu$. So take $\lambda=\sqrt{A-C}$ and $\mu=\operatorname{sign}(D) \sqrt{B-C}$.

Proofs of case 2 and case 3 are similar to that of case 1

The following proposition deals with the general case.

Proposition (4.3):

For the circular cone $A x^{2}+B y^{2}+C z^{2}+D x y+E x z+F y z+G x+H y+I z+J=0$ with axis direction $\left.\lambda_{v} \quad \mu_{v} \quad v\right)$, let $D(0, E(0, F(0)$ and $D E F>0$ then

$\lambda=\operatorname{sign}(F) \sqrt{\frac{D E}{2 F}}, \mu=\operatorname{sign}(E) \sqrt{\frac{D F}{2 E}}, v=\operatorname{sign}(D) \sqrt{\frac{E F}{2 D}}$. 
If $D E F \ngtr 0$ then the cone's equation is multiplied by (-1) and the resulting coefficients are used.

\section{Proof:}

Equations (7-9) give $=\mathbf{2} \lambda \mu, \quad E=\mathbf{2} \lambda v, \quad F=2 \mu v$

Solving these equations for $\lambda, \mu$, and $\nu$, the result is

$\lambda^{2}=\frac{D E}{2 F}, \quad \mu^{2}=\frac{D F}{2 E}, \quad v^{2}=\frac{E F}{2 D}$

This leads to $\lambda= \pm \sqrt{\frac{D E}{2 F}}, \quad \mu= \pm \sqrt{\frac{D F}{2 E}}, \quad v= \pm \sqrt{\frac{E F}{2 D}}$.

The signs are chosen so that equations (7-9) are satisfied. The following table solves the problem of signs.

Note that $D E F>0 \Rightarrow \operatorname{sign}(D) \operatorname{sign}(E) \operatorname{sign}(F)=+$

Table (4.1): Relations between signs

\begin{tabular}{|c|c|c|c|c|c|}
\hline $\operatorname{sign}(D)$ & $\operatorname{sign}(E)$ & $\operatorname{sign}(F)$ & $\operatorname{sign}(D) \operatorname{sign}(E)$ & $\operatorname{sign}(D) \operatorname{sign}(F)$ & $\operatorname{sign}(E) \operatorname{sign}(F)$ \\
\hline+ & + & + & + & + & + \\
\hline+ & - & - & - & - & + \\
\hline- & + & - & - & + & - \\
\hline- & - & + & + & - & - \\
\hline
\end{tabular}

Setting $\lambda=\operatorname{sign}(F) \sqrt{\frac{D E}{2 F}}, \quad \mu=\operatorname{sign}(E) \sqrt{\frac{D F}{2 E}}, \quad v=\operatorname{sign}(D) \sqrt{\frac{E F}{2 D}} \Rightarrow$

$D=\mathbf{2} \lambda \mu, E=\mathbf{2} \lambda \nu, \quad F=\mathbf{2} \mu \nu$ as required

\section{Extracting the vertex semi-angle $\alpha$}

The semi-angle of the vertex is obtained using equation (4-6), that is, by using any of the following formulas

$$
\begin{aligned}
& \cos ^{2} \alpha=\frac{\lambda^{2}-A}{\lambda^{2}+\mu^{2}+\nu^{2}} \\
& \cos ^{2} \alpha=\frac{\mu^{2}-B}{\lambda^{2}+\mu^{2}+\nu^{2}} \\
& \cos ^{2} \alpha=\frac{\nu^{2}-C}{\lambda^{2}+\mu^{2}+\nu^{2}}
\end{aligned}
$$

All the above forms should give the same result. If they are not equal, multiply the cone's equation by (-1) and use the obtained coefficients. If also they are not equal then the surface is not a right circular cone.

\section{The Algorithm}

\section{START}

'Step 1: Input the coefficients of the cone's algebraic equation

INPUT A, B, C, D, E, F, G, H, I, J

' Step 2: Extracting the cone vertex $\left(x_{a}, y_{a} z_{n}\right)$

$\Delta=\left|\begin{array}{ccc}2 A & D & E \\ D & 2 B & F \\ E & F & 2 C\end{array}\right|$ 
IF $\boldsymbol{\Delta}=\mathbf{0}$ THEN OUTPUT "not a cone" and STOP

ELSE IF $\Delta \neq 0$ THEN

$$
\begin{gathered}
\mathbf{\Delta}_{\mathbf{1}}=\left|\begin{array}{ccc}
G & D & E \\
-H & 2 B & F \\
-I & F & 2 C
\end{array}\right|: \quad \mathbf{\Delta}_{\mathbf{2}}=\left|\begin{array}{ccc}
2 A & -G & E \\
D & -H & F \\
E & -I & 2 C
\end{array}\right|: \quad \mathbf{\Delta}_{\mathbf{2}}=\left|\begin{array}{ccc}
2 A & D & -G \\
D & 2 B & -H \\
E & F & -I
\end{array}\right| \\
x_{0}=\frac{\Delta_{1}}{\Delta} \quad: \quad y_{0}=\frac{\Delta_{2}}{\Delta} \quad: \quad z_{0}=\frac{\Delta_{\mathrm{g}}}{\mathbf{\Delta}}
\end{gathered}
$$

ENDIF

' Check

IF $l \neq A x_{0}^{2}+B y_{0}^{2}+C z_{0}^{z}+D x_{0} y_{0}+E x_{0} z_{0}+F y_{0} z_{0}$

THEN OUTPUT " not a cone" and STOP

' Step 3: Extracting the axis direction vector $\boldsymbol{n}_{s} \quad \mu_{s} \quad v$ )

$$
\lambda=0: \mu=0: v=0
$$

FOR $\mathrm{k}=1,2$

$\mathrm{IF} D=E=F=0$ AND $A B C>0$ AND $C>0$ AND $A=B$

\section{THEN}

OUTPUT "the cone axis is parallel to the z-axis"

$$
\lambda=0: \mu=0: v=\sqrt{C-A}
$$

ELSE IF $D=E=F=0$ AND $A B C>0$ AND $B>0$ AND $A=C$

THEN

OUTPUT "the cone axis is parallel to the y-axis"

$$
\lambda=0: \mu=\sqrt{B-C}: v=0
$$

ELSE IF $D=E=F=0$ AND $A B C>0$ AND $A>0$ AND $B=C$

THEN

OUTPUT "the cone axis is parallel to the $\mathrm{x}$-axis"

$$
\lambda=\sqrt{A-C}: \mu=0: v=0
$$

ELSEIF $D \neq 0$ AND $E=F=0$ AND $C<0$

THEN

OUTPUT "the cone axis is parallel to the xy-plane"

$$
\lambda=\sqrt{A-C}: \mu=\operatorname{sign}(D) \sqrt{B-C}: \nu=0
$$

ELSEIF $D=F=0$ AND $E \neq 0$ AND $B<0$

THEN

OUTPUT "the cone axis is parallel to the xz-plane"

$$
\lambda=\sqrt{A-B}: \mu=0: v=\operatorname{sign}(E) \sqrt{C-B}
$$

ELSEIF $D=E=0$ AND $F \neq 0$ AND $A<0$

THEN

OUPUT "the cone axis is parallel to the yz-plane"

$$
\lambda=0: \mu=\sqrt{B-A}: v=\operatorname{sign}(F) \sqrt{C-A}
$$

ELSEIF $D E F>0$

THEN 


$$
\lambda=\operatorname{sign}(F) \sqrt{\frac{D E}{2 F}}, \mu=\operatorname{sign}(E) \sqrt{\frac{D F}{2 E}}: v=\operatorname{sign}(D) \sqrt{\frac{E F}{2 D}}
$$

$\operatorname{ELSE} A=-A: B=-B: C=-C: D=-D: E=-E: F=-F$

ENDIF

NEXT k

IF $\lambda=\mu=v=0$ THEN OUTPUT "not a cone" and STOP

' Step 4: Extracting the vertex semi-angle $\alpha$

IF $\lambda^{2}-A=\mu^{2}-B=v^{2}-C$ THEN $\alpha=\cos ^{-1} \sqrt{\frac{\lambda^{2}-A}{\lambda^{2}+\mu^{2}+v^{2}}}$

ELSEIF $\lambda^{2}+A=\mu^{2}+B=v^{2}+C$ THEN $\alpha=\cos ^{-1} \sqrt{\frac{\lambda^{2}+A}{\lambda^{2}+\mu^{2}+v^{2}}}$

ELSE OUTPUT " not a right circular cone" and STOP

ENDIF

'Step 5: Output the cone's geometric parameters

OUTPUT $x_{0}, y_{0}, z_{0}, \lambda, \mu, v, \alpha$

STOP

\section{Test Examples}

7.1. $x^{2}-y^{2}-z^{2}=0$.

$$
\begin{aligned}
& A=1, B=-1, C=-1, D=E=F=G=H=I=J=0 . \\
& \Delta=8 \neq 0, \Delta_{1}=\Delta_{2}=\Delta_{2}=0 \Rightarrow x_{0}=y_{0}=z_{0}=0 . \\
& I=0+0+0+0+0+0=0 \text { O.K. }
\end{aligned}
$$

$A B C>0, B=C, A>0 \Rightarrow$ the cone-axis is parallel to $x$-axis

$$
\begin{gathered}
\lambda=\sqrt{2}, \mu=0, v=0 \\
\lambda^{2}-A=\mu^{2}-B=v^{2}-C=1 \Rightarrow \text { O.K. }
\end{gathered}
$$

$\alpha=\cos ^{-1} \sqrt{\frac{1}{2}}=45^{\circ}$

7.2. $x^{2}+y^{2}-z^{2}=0$.

$$
\begin{aligned}
& A=B=1, C=-1, D=E=F=G=H=I=J=0 . \\
& \Delta=-8 \neq 0, \Delta_{1}=\Delta_{2}=\Delta_{3}=0 \Rightarrow x_{0}=y_{0}=z_{0}=0 .
\end{aligned}
$$

The conditions $A B C>0$ and $C>0$ are not satisfied, so the cone's equation is multiplied by (-1) giving $-x^{2}-y^{2}+z^{2}=0$, then

$$
A=B=-1, C=1 \text {. }
$$

$\therefore$ The cone axis is parallel to $z$-axis.

$$
\begin{gathered}
\lambda=\mu=0, \quad v=\sqrt{2} . \\
\alpha=\cos ^{-1} \sqrt{\frac{1}{2}}=45^{\circ} .
\end{gathered}
$$

7.3. $-3 x^{2}-3 y^{2}+z^{2}+6 x-6 y-6=0$ 


$$
\begin{aligned}
& A=-3, B=-3, C=1, D=E=F=0, G=6, H=-6, I=0, I=-6 . \\
& \mathrm{C}=72, \mathrm{G}_{1} 1=72, \mathrm{G}_{1}=-72, \mathrm{G}^{3}=0 \Rightarrow x_{0}=1, y_{0}=-1, z_{0}=0 . \\
& I=-3-3+0+0+0+0=-6 \text { O.K. } \\
& D=E=F=0,>0, C>0 \text { and } A=B
\end{aligned}
$$

Then the cone-axis is parallel to $\mathrm{z}$-axis.

$$
\begin{aligned}
& \lambda=0: \mu=0: v=\sqrt{1+3}=2 \\
& \lambda^{2}-A=\mu^{2}-B=v^{2}-C=3 \text { O.K. } \\
& \alpha=\cos ^{-1} \sqrt{\frac{3}{4}}=30^{\circ} .
\end{aligned}
$$

7.4. $11 x^{2}-y^{2}+15 z^{2}-16 x y=0$.

$$
\begin{aligned}
& A=11, B=-1, C=15, D=-16, E=F=G=H=I=J=0 . \\
& \Delta=-1320 \neq 0, \Delta_{1}=\Delta_{2}=\Delta_{3}=0 \Rightarrow x_{0}=y_{0}=z_{0}=0 .
\end{aligned}
$$

The condition $C<0$ is not satisfied, so the cone's equation is multiplied by (-1) giving $-11 x^{2}+y^{2}-15 z^{2}+16 x y=0$, then

$A=-11, B=1, C=-15, D=16, E=F=G=H=I=J=0$.

The cone axis is parallel to the $x y$-plane.

$\lambda=\sqrt{-11+15}=2, \mu=\sqrt{1+15}=4, v=0$.

$\lambda^{2}-A=\mu^{2}-B=v^{2}-C=15$ O.K.

Then,

$$
\alpha=\cos ^{-1} \sqrt{\frac{15}{20}}=30^{\circ}
$$

7.5. $3 x^{2}+3 y^{2}-z^{2}-8 x y+8 x-6 y-2 z+2=0$.

$$
\begin{aligned}
& A=3, B=3, C=-1, D=-8, E=F=0, G=8, H=-6, I=-2, J=2 \text {. } \\
& \mathrm{c}=-72, \mathrm{G}_{1} 1=0, \mathrm{G}_{1} 2=-72, \mathrm{G}_{3} 3=72 \Rightarrow x_{0}=0, y_{0}=1, z_{0}=-1 \text {. } \\
& I=0+3-1+0+4+0=2 \text { O.K. } \\
& D \neq 0, E=F=0 \text { and } C<0 \Rightarrow \text { the cone-axis is parallel to } x y \text {-plane. } \\
& \lambda=2, \mu=-2, v=0 \\
& \lambda^{2}-A=\mu^{2}-B=v^{2}-C=1 \text { O.K. } \\
& \alpha=\cos ^{-1} \sqrt{\frac{1}{8}} \approx 69.3^{\circ}
\end{aligned}
$$

7.6. $y^{2}+2 x z=0$.

$$
\begin{aligned}
& B=\mathbf{1}, E=2, A=C=D=F=H=G=I=J=0 \\
& \mathbf{l}=-\mathbf{8}, \mathbf{G}_{1} 1=\mathrm{G}_{1} 2=\mathrm{G}_{1} 3=0 \Rightarrow x_{0}=y_{0}=z_{0}=0 .
\end{aligned}
$$

$E$ ( 0 but $B<0$ is not satisfied, so multiply the cone's equation by (-1) giving

$$
\begin{gathered}
-y^{2}-2 x z=0 \Rightarrow B=-1, E=-2, \\
A=C=D=F=H=G=I=J=0 .
\end{gathered}
$$

Then, the cone axis is parallel to the $x z$-plane.

$$
\begin{gathered}
\lambda=1, \mu=0, \nu=-1 \\
\lambda^{2}-A=\mu^{2}-B=v^{2}-C=1 \text { O.K. } \\
\alpha=\cos ^{-1} \sqrt{\frac{1}{2}}=45^{\circ}
\end{gathered}
$$




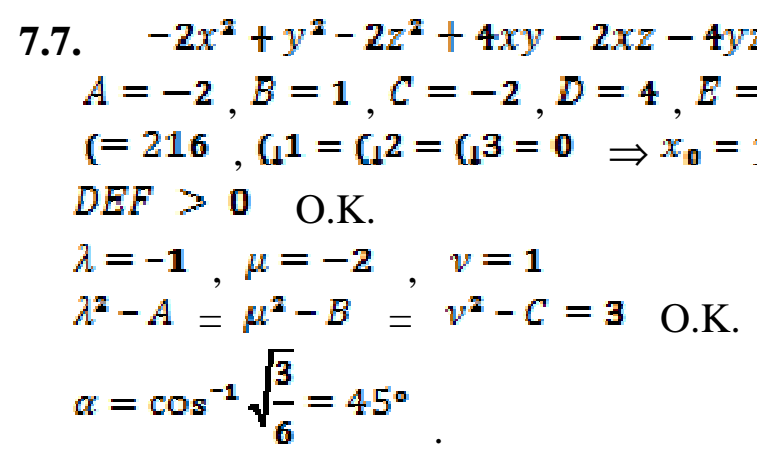

7.8. $x^{2}-2 y^{2}-2 z^{2}+4 x y-4 x z-2 y z-6 x-6 y+3=0$.

$$
\begin{aligned}
& A=1, B=-2, C=-2, D=4, E=-4, F=-2, G=-6, H=-6, I=0, I=3 \text {. } \\
& \mathrm{C}=216, \mathrm{G}_{1} 1=216, \mathrm{G}_{1} 2=0, \mathrm{G}_{1} 3=-216 \Rightarrow x_{0}=1, y_{0}=0, z_{0}=-1 \text {. } \\
& I=1+0-2+0+4+0=3 \text { O.K. } \\
& D E F>0 \text { O.K. } \\
& \lambda=-2: \mu=-1: v=1 \\
& \lambda^{2}-A=\mu^{2}-B=v^{2}-C=3 \quad \text { O.K. } \\
& \alpha=\cos ^{-1} \sqrt{\frac{3}{6}}=45^{\circ}
\end{aligned}
$$

7.9. $x y+x z+y z=0$.

$$
\begin{aligned}
& A=B=C=0, D=E=F=1, G=H=I=J=0 . \\
& \left(=2,{ }_{1} 1=0, \mathrm{G}^{2}=0, \mathrm{G}^{3}=0 \Rightarrow x_{0}=0, y_{0}=0, z_{0}=0 .\right. \\
& I=0+0+0+0+0+0=0 \text { O.K. } \\
& D E F>0 \text { O.K. }
\end{aligned}
$$$$
\lambda=\frac{1}{\sqrt{2}}, \mu=\frac{1}{\sqrt{2}}, \quad v=\frac{1}{\sqrt{2}}
$$$$
\lambda^{2}-A=\mu^{2}-B=v^{2}-C=0.5 \quad \text { O.K. }
$$$$
\alpha=\cos ^{-1} \sqrt{\frac{0.5}{1.5}} \approx 54.74^{\circ}
$$

7.10. Not a Cone Case

$$
\begin{aligned}
& 6 x^{2}+35 y^{2}+24 z^{2}-29 x y+24 x z-58 y z=0 . \\
& A=6, B=35, C=24, D=-29, E=24, F=-58, \\
& G=H=I=J=0 . \\
& (=0 \Rightarrow \text { Not a Cone. }
\end{aligned}
$$

7.11. Not a Right Circular Cone Case

$-2 y^{2}+8 x y+4 x z+8 y z-6 x+4 y+2 z-5=0$.

$A=0, B=-2, C=0, D=8, E=4, F=8, G=-6, H=4, I=2, l=-5$.

$\mathrm{c}=576, \mathrm{G}_{1} 1=672, \mathrm{G}_{1} 2=-192, \mathrm{G}_{1} 3=-480 \Rightarrow x_{0}=\frac{7}{6}, y_{0}=-\frac{1}{3}$,

$z_{0}=-\frac{5}{6}$

$J=0+\left(-\frac{2}{9}\right)+0+\left(-\frac{56}{18}\right)+\left(-\frac{140}{36}\right)+\left(\frac{40}{18}\right)=-5$

$D E F>0$ O.K. 


$$
\begin{gathered}
\lambda=\sqrt{2}, \mu=2 \sqrt{2}, \quad v=\sqrt{2} \\
\lambda^{2}-A=2, \quad \mu^{2}-B=10, \quad v^{2}-C=2 \Rightarrow \text { Not a Right Circular Cone. }
\end{gathered}
$$

\section{CONCLUSION}

The right circular cone is an important geometric primitive that appears in many applications and still live in many research directions. The geometric parameters of the right circular cone are the vertex coordinates, the axis direction, and the vertex semi-angle. These parameters are hidden in the coefficients of the algebraic equation of the cone. In this paper an algorithm is introduced for extracting these parameters from the coefficients of the algebraic equation. A solid theoretical ground is set through three propositions. The first proposition is concerned with right circular cones whose axes are parallel to one of the principal axes. The second proposition is concerned with right circular cones whose axes are parallel to one of the principal planes. The third proposition is concerned with right circular cones whose axes are in general positions. The algorithm is tested on eleven cases and proves reliability and simplicity. The algorithm can also detect non right circular cone cases.

\section{REFERENCES}

1. [ Afwat, M., 1978], Geometry part II, pp. 143, Zagazig University, Zagazig, Egypt.

2. [Andrews, J. and Séquin, C.H., 2013], Type-Constrained Direct Fitting of Quadric Surfaces, Computer-Aided Design \& Applications, Vol.10, pp. 1-15.

3. [Busé, L. et al.,2016], Extraction of cylinders and cones from minimal point sets, Graphical Models, Elsevier, Vol. 86, pp.1-12.

4. [Ilyin, V.A. and Poznyak, E.G., 1984], Analytic Geometry, pp. 191, Mir Publishers, Moscow.

5. [Liu, Y. et al., 2006], Geometric Modeling with Conical Meshes and Developable Surfaces, ACM Transactions on Graphics, Vol. 25, No. 3, pp. 681-689.

6. [Lukács, G. et al., 1997], Geometric Least-Squares Fitting of Spheres, Cylinders, Cones and Tori, Technical Report; Department of Computer Science, University of Wales: Cardiff, UK.

7. [Lukacs, G. et al., 1998], Faithful Least-Squares Fitting of Spheres, Cylinders, Cones and Tori for Reliable Segmentation, In: Proceedings of $5^{\text {th }}$ European Conference on Computer Vision (ECCV'98), pp. 671-686, Freiburg, Germany.

8. [Mortenson, M.E., 1990], Computer Graphics Handbook: Geometry and Mathematics, pp. 167, Industrial Press Inc., New York.

9. [Mulchrone, K. F. et al., 2013], Mathematica code for least-squares cone fitting and equal-area stereonet representation, Computers \& Geosciences, Vol. 54, pp. 203-210.

10. [Pogorelov, A.V., 1980], Analytic Geometry, pp. 154, Mir Publishers, Moscow.

11. [Salilew, G.A., 2017], New Approach on Identification of Circular Cone, Mathematical Theory and Modeling, Vol.7, No.8.

12. [Toony, Z., et al., 2015], PGP2X: Principal Geometric Primitives Parameters Extraction, In: Proceedings of the $10^{\text {th }}$ International Joint Conference on Computer Vision, Imaging and Computer Graphics Theory and Applications (VISIGRAPP2015), Berlin, Germany.

13. [Zhang, L. and Guo, C., 2018], A method for cone fitting based on certain sampling strategy in CMM metrology, AIP Conference Proceedings 1955, 040174. 